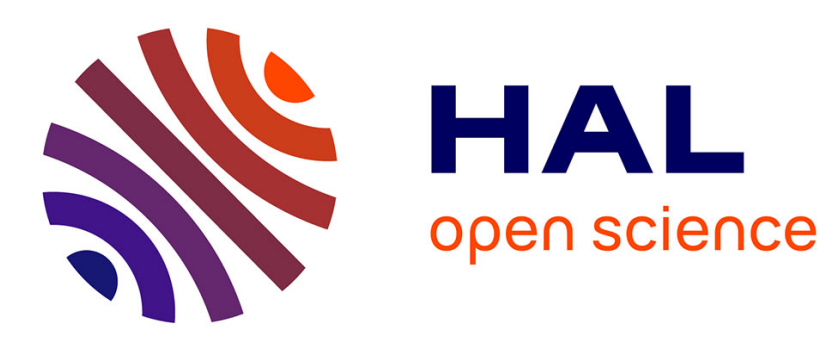

\title{
A structured approach for input derivative index-2 descriptor dynamical model control
}

Charles Poussot-Vassal, Pierre Vuillemin, David Quero

\section{To cite this version:}

Charles Poussot-Vassal, Pierre Vuillemin, David Quero. A structured approach for input derivative index-2 descriptor dynamical model control. ECC 2019, Jun 2019, NAPLES, Italy. pp.4258-4263, 10.23919/ECC.2019.8795953 . hal-02335102

\section{HAL Id: hal-02335102 https://hal.science/hal-02335102}

Submitted on 28 Oct 2019

HAL is a multi-disciplinary open access archive for the deposit and dissemination of scientific research documents, whether they are published or not. The documents may come from teaching and research institutions in France or abroad, or from public or private research centers.
L'archive ouverte pluridisciplinaire HAL, est destinée au dépôt et à la diffusion de documents scientifiques de niveau recherche, publiés ou non, émanant des établissements d'enseignement et de recherche français ou étrangers, des laboratoires publics ou privés. 


\title{
A structured approach for input derivative index-2 descriptor dynamical model control
}

\author{
C. Poussot-Vassal, P. Vuillemin and D. Quero
}

\begin{abstract}
This paper reveals a practical manner to deal with the control design of dynamical systems governed by input derivative index-2 first order differential algebraic equations. This class of dynamical model embeds a polynomial which results in a infinite gain in high frequencies. The idea is grounded on the construction of an adequate SISO controller structure to make possible the control design on a model presenting both rational and a first order polynomial parts. The design approach then leads to a controller in a rational form without any algebraic constrain, which can be implemented on a real-time target. Application for gust load control of a complex aeroservoelastic aircraft dynamical model, which naturally embeds an input derivative action, illustrates the approach, using an $\mathcal{H}_{\infty}$-norm minimization performance objective.
\end{abstract}

\section{INTRODUCTION}

\section{A. Motivating context and problem formulation}

Let us be given $\mathbf{H} \notin \mathcal{L}_{\infty}$, an $n$-th order $n_{u}$ inputs $(\mathbf{u}) n_{y}$ outputs (y) linear time invariant complex-valued dynamical model defined as:

$$
\begin{aligned}
\mathbf{y}(s) & =\mathbf{H}(s) \mathbf{u}(s) \\
& =\mathbf{H}_{r}(s) \mathbf{u}(s)+s \mathbf{H}_{r}(s) \mathbf{u}(s) \\
& =\left(\mathbf{H}_{r}(s)+\mathbf{H}_{p}(s)\right) \mathbf{u}(s) \\
& =\left(C(s E-A)^{-1} B+D\right) \mathbf{u}(s) .
\end{aligned}
$$

where $s$ denotes the Laplace variable. We denote the rational part of $\mathbf{H}$ as $\mathbf{H}_{r} \in \mathcal{L}_{\infty}$ and the polynomial one as $\mathbf{H}_{p} \notin \mathcal{L}_{\infty}$. Transfer (1) admits a descriptor realization $\mathcal{S}:(E, A, B, C, D)$ given as

$$
\mathcal{S}:\left\{\begin{aligned}
E \dot{\mathbf{x}}(t) & =A \mathbf{x}(t)+B \mathbf{u}(t) \\
\mathbf{y}(t) & =C \mathbf{x}(t)+D \mathbf{u}(t)
\end{aligned}\right.
$$

where $\mathbf{x}(t) \in \mathbb{R}^{n}, \mathbf{u}(t) \in \mathbb{R}^{n_{u}}$ and $\mathbf{y}(t) \in \mathbb{R}^{n_{y}}$ represent the internal variables, input and output vectors, respectively. Then, $E, A \in \mathbb{R}^{n \times n}, B \in \mathbb{R}^{n \times n_{u}}, C \in \mathbb{R}^{n_{y} \times n}$ and $D \in$ $\mathbb{R}^{n_{u} \times n_{y}}$. Due to the $\mathbf{u}(s)$ input and input derivative impacts in (1), (2) is a DAE for differential algebraic equations, embedding a polynomial part and a rank deflecting $E$ matrix. By admitting the canonical form transformation, (2) may be rewritten as (see e.g. [1]),

$$
\overline{\mathcal{S}}:\left\{\begin{aligned}
\bar{E}\left[\begin{array}{c}
\dot{\mathbf{x}}_{1}(t) \\
\dot{\mathbf{x}}_{2}(t)
\end{array}\right] & =\bar{A}\left[\begin{array}{c}
\mathbf{x}_{1}(t) \\
\mathbf{x}_{2}(t)
\end{array}\right]+\bar{B} \mathbf{u}(t) \\
\mathbf{y}(t) & =\bar{C}\left[\begin{array}{c}
\mathbf{x}_{1}(t) \\
\mathbf{x}_{2}(t)
\end{array}\right]
\end{aligned}\right.
$$

C. Poussot-Vassal and P. Vuillemin are with Onera - The French Aerospace Lab, F-31055 Toulouse, France; email: charles.poussotvassal@onera.fr, pierre.vuillemin@onera.fr

D. Quero is with DLR, Institute of Aeroelasticity, 37073, Gottingen, Germany; email: david.queromartin@dlr.de where

$$
\begin{gathered}
\bar{E}=\left[\begin{array}{cc}
I_{n_{1}} & 0 \\
0 & N
\end{array}\right], \bar{A}=\left[\begin{array}{cc}
A_{1} & 0 \\
0 & I_{n_{2}}
\end{array}\right] \\
\bar{B}=\left[\begin{array}{l}
B_{1} \\
B_{2}
\end{array}\right], \bar{C}=\left[\begin{array}{ll}
C_{1} & C_{2}
\end{array}\right],
\end{gathered}
$$

and where $N \in \mathbb{R}^{n_{2} \times n_{2}}$ is a $k$-nilpotent matrix, i.e. $N^{k}=$ $0_{n_{2}}$. Following equation (3), let $\mathbf{x}_{1}(t) \in \mathbb{R}^{n_{1}}, \mathbf{x}_{2}(t) \in$ $\mathbb{R}^{n_{2}}$ and $r=\operatorname{rank}(E)$. Then, $n_{1}$ denotes the number of finite dynamic modes, $r-n_{1}=\operatorname{rank}(N)$ the infinite dynamic (impulsive) modes and $n-r$, the non-dynamic modes. The index of the DAE is given by the number of differentiation needed to get an ODE (ordinary differential equation), e.g. equation with no impulsive and non-dynamic modes. Given the canonical realization as in (3), the index is the degree of nilpotency $k$ of matrix $N$. Moreover, we also denote as $\mathcal{S}_{r}$, the realization associated to $\mathbf{H}_{r} \in \mathcal{L}_{\infty}$, and is given as

$$
\mathcal{S}_{r}:\left\{\begin{aligned}
E_{r} \dot{\mathbf{x}}_{r}(t) & =A_{r} \mathbf{x}_{r}(t)+B_{r} \mathbf{u}(t) \\
\mathbf{y}_{r}(t) & =C_{r} \mathbf{x}_{r}(t)+D_{r} \mathbf{u}(t)
\end{aligned}\right.
$$

where $\mathbf{x}_{r}(t) \in \mathbb{R}^{n_{r}}$, with $n_{r}<n$ and where $E_{r}, A_{r}, B_{r}, C_{r}, D_{r}$ are of appropriate dimensions and with $\left(E_{r}, A_{r}\right)$ pencil regular.

In this paper we will consider a polynomial part $\mathbf{H}_{p}(s)=$ $s \mathbf{H}_{r}(s)$, i.e. as in (1). Moreover, given the canonical realization of $\mathbf{H}$, a nilpotent matrix $N$ of order $k=2$ is considered. As a consequence, the DAE the and the $(E, A)$ matrix pencil presents some infinite eigenvalues, and thus, $\operatorname{rank}(E)=r<n$. In this case, the model is said to be DAE and descriptor of index $-2^{1}$.

Due to this specific (1)-(3) formulation, the transfer $\mathbf{H}(s)$ does not belong to the rational complex meromorphic function space of systems with finite energy any longer. As a matter of consequence, the standard $\mathcal{H}_{\infty}$-norm control oriented methods do not apply anymore, and specific methods must be applied. Let us anyway formulate, with a slight abuse of language, the considered problem in what follows.

Design a rational $n_{K}$-th order controller $\mathbf{K}^{\star} \in \mathcal{H}_{\infty}$, which ensures closed-loop stability and minimizes some Hardy norm performance objective, solving the following problem:

$$
\mathbf{K}^{\star}:=\arg \min _{\mathbf{K} \in \mathcal{K} \subseteq \mathcal{H}_{\infty}}\left\|\mathcal{F}_{l}(\mathbf{G}(\mathbf{H}), \mathbf{K})\right\|_{\mathcal{H}_{\infty}}
$$

${ }^{1}$ Throughout this paper, we denote $\mathcal{H}_{2}$ (resp. $\mathcal{H}_{\infty}$ ), the open subspace of $\mathcal{L}_{2}$ (resp. $\mathcal{L}_{\infty}$ ) with matrix-valued function $\mathbf{H}(s)$ with $n_{y}$ outputs, $n_{u}$ inputs, $\forall s \in \mathbb{C}$, which are analytic in $\operatorname{Re}(s)>0$ (resp. $\operatorname{Re}(s) \geq 0$ ). Moreover $\mathcal{H}_{2}$ functions integral along the imaginary axis are bounded and $\mathcal{H}_{\infty}$ ones have a finite supremum on this axis (see [2], [3]). 
where $\mathcal{F}_{l}(.,$.$) denotes the lower linear fractional operators$ (see [4] for details on this notation) and where we denote $\mathbf{K} \in \mathcal{K} \subseteq \mathcal{H}_{\infty}$ the controller rational function equipped with the ODE realization $\mathcal{S}_{K}:\left(I_{n_{K}}, A_{K}, B_{K}, C_{K}, D_{K}\right)$. Moreover, the generalized dynamical model is given as

$$
\mathbf{G}(\mathbf{H})(s)=\mathbf{W}_{i}(s) \mathbf{H}(s) \mathbf{W}_{o}(s)
$$

where $\mathbf{W}_{i}(s)$ and $\mathbf{W}_{o}(s)$ are the input and output weighting functions, classically used in any $\mathcal{H}_{\infty}$ control problem to weight the performance transfer (see e.g. [5]). Obviously, we consider that $\mathbf{G}(\mathbf{H})$ has a similar structure as (2), and thus, $\mathbf{G}(\mathbf{H}) \notin \mathcal{L}_{\infty}$.

\section{B. Contributions and outlines}

The objective of this paper is to provide a simple solution for designing of a linear dynamical controller described as a reduced-order ordinary differential equation set (ODE), solving (6). More specifically, the main idea is to exploit the specific DAE structure of (1)-(3), in order to transform the original model (1)-(3) into a rational one on which problem (6) is applicable and appropriate for Hardy-norm minimization. Based on this reformulation, and thanks to the available structured $\mathcal{H}_{\infty}$-norm oriented optimization tools made available in MATLAB through the hinfstruct method [6], we provide a simple but yet effective way to deal with this problem in a simple practical way. The proposed approach is applied on a complex aeroservoelastic aircraft model modeling the flight dynamics, aeroelastic modes, delay and dynamical loads effects, subject to vertical gust disturbances (see e.g. [7], [8]). Such a use-case, which is largely exploited by aeroelastical and aerodynamical industrial and research engineers, explicitly embeds the input derivative in the equations, as in (1), leading to an index-2 descriptor model. Controlling such a model is very challenging in view of aircraft load and consumption reductions. Note that in the traditional works, the high frequency derivative action embedded in $\mathbf{H}_{p}$ (and infinite eigenvalues) are neglected / removed to stick to standard tools. However, this approximation is mathematically incorrect since it arbitrarily removes impulsive dynamics. This is the reason why, here instead, the proposed approach directly attacks the problem with no approximation.

The remaining of the paper is organized as follows: the main result, i.e. the structured approach for index-2 descriptor model with input derivative linear controller synthesis is presented in Section II. The application on the complex aeroelastic aircraft model is done in Section III. Discussions close the paper in Section IV.

It is to be mentioned that solutions dealing with the general case of DAE control have been treated in the literature to address somehow (6). Among them, [9] proposed a linear matrix inequality based approach for $\mathcal{H}_{\infty}$ control design, leading to a solution for the case where $\mathbf{K}^{\star}$ is of full order. One may also mention [10] which involves the Riccati equations and conditions on the observability and detectability to address a larger DAE class. Similarly, one limitation is related to the structure of the controller, which may increase with the model complexity. Still, to the authors best of knowledge, a few (if none) did treat such a problem using a dedicated structure tailored to the considered usecase.

\section{MAIN RESUlT: STRUCTURED APPROACH FOR INDEX-2 DESCRIPTOR LINEAR CONTROLLER SYNTHESIS}

\section{A. Classical problem formulation with $\mathcal{H}_{\infty}$ performances}

Let us consider a DAE linear dynamical model of the form (1)-(3). As evoked in the introductory part, we aim at designing rational controller that ensures some $\mathcal{H}_{\infty}$ performances and which applies on such index-2 DAE model. As is standard in the robust framework, let us first define the following generalized plant $\mathbf{G}(s)=\mathbf{W}_{i}(s) \mathbf{H}(s) \mathbf{W}_{o}(s)$, where, $\mathbf{W}_{i}(s)$ and $\mathbf{W}_{o}(s)$ are the weighting filters defining the shaped input and output signals ${ }^{2}$. Following the notations given in Section I, the associated state-space realization of $\mathbf{G}(\mathbf{H})$ is then given by,

$$
\left\{\begin{aligned}
E^{G} \dot{\mathbf{x}}^{G}(t) & =A^{G} \mathbf{x}^{G}(t)+B_{1}^{G} \mathbf{w}(t)+B_{2}^{G} \mathbf{u}(t) \\
\mathbf{z}(t) & =C_{1}^{G} \mathbf{x}^{G}(t)+D_{11}^{G} \mathbf{w}(t)+D_{12}^{G} \mathbf{u}(t) \\
\mathbf{y}(t) & =C_{2}^{G} \mathbf{x}^{G}(t)+D_{21}^{G} \mathbf{w}(t)+D_{22}^{G} \mathbf{u}(t)
\end{aligned}\right.
$$

where $\mathbf{x}^{G}(t) \in \mathbb{R}^{n+n_{W}}$ (where $n_{W}$ is the dimension of the weighting transfer $\mathbf{W}_{i}(s)$ and $\left.\mathbf{W}_{o}(s)\right), \mathbf{w}(t) \in \mathbb{R}^{n_{w}}, \mathbf{u}(t) \in$ $\mathbb{R}^{n_{u}}, \mathbf{z}(t) \in \mathbb{R}^{n_{z}}$ and $\mathbf{y}(t) \in \mathbb{R}^{n_{y}}$ are the states, exogenous input, control input, performance output and measurement signals, respectively. The $G$ subscript matrices are of appropriate dimension and function of (3) and $\mathbf{W}_{i}(s)$ and $\mathbf{W}_{o}(s)$. However, at this point, we consider that the generalized model still is descriptor and embeds a polynomial part as in (2) or (3) (indeed, the weight shall not modify this stricture). To alleviate this limitation, next, we take advantage of the problem structure to define an equivalent modified problem.

\section{B. Input augmented dynamical model and problem formula- tion with $\mathcal{H}_{\infty}$ performances}

If now, instead of considering (1)-(3) to design the generalized plant, we consider the following input augmented model $\tilde{\mathbf{H}} \in \mathcal{L}_{\infty}$ with realization $\tilde{\mathcal{S}}$, which directly comes from the rational part (5) of (2),

$$
\tilde{\mathcal{S}}:\left\{\begin{aligned}
E_{r} \dot{\mathbf{x}}(t) & =A_{r} \mathbf{x}_{r}(t)+\left[\begin{array}{ll}
B_{r} & B_{r}
\end{array}\right]\left[\begin{array}{c}
\mathbf{u}(t) \\
\mathbf{u}_{p}(t)
\end{array}\right] \\
\mathbf{y}(t) & =C_{r} \mathbf{x}_{r}(t)+\left[\begin{array}{ll}
D_{r} & D_{r}
\end{array}\right]\left[\begin{array}{c}
\mathbf{u}(t) \\
\mathbf{u}_{p}(t)
\end{array}\right]
\end{aligned}\right.
$$

which is no longer descriptor (i.e. $\left(E_{r}, A_{r}\right)$ pencil regular), but which involves an additional input $\mathbf{u}_{p}(t)$ that must satisfy

$$
\mathbf{u}_{p}(t)=\dot{\mathbf{u}}(t)
$$

i.e. be exactly the input derivative of $\mathbf{u}(t)^{3}$. This manipulation is possible due to the particular structure of the

\footnotetext{
${ }^{2}$ Both $\mathbf{W}_{i}(s)$ and $\mathbf{W}_{o}(s)$ may be constructed by the user to define the desired performances attenuation and its bandwidth

${ }^{3}$ Note that $\tilde{\mathbf{H}}$ is a copy of $\mathbf{H}$, with an additional input.
} 
original model (1). Then, the generalized plant $\mathbf{G}(\tilde{\mathbf{H}}) \in \mathcal{L}_{\infty}$ associated realization is now given as

$$
\left\{\begin{aligned}
E^{g} \dot{\mathbf{x}}^{g}(t) & =A^{g} \mathbf{x}^{g}(t)+B_{1}^{g} \mathbf{w}(t) \\
& +\left[\begin{array}{ll}
B_{2}^{g} & B_{2}^{g}
\end{array}\right]\left[\begin{array}{c}
\mathbf{u}(t) \\
\mathbf{u}_{p}(t)
\end{array}\right] \\
\mathbf{z}(t) & =C_{1}^{g} \mathbf{x}^{g}(t)+D_{11}^{g} \mathbf{w}(t) \\
& +\left[\begin{array}{ll}
D_{12}^{g} & D_{12}^{g}
\end{array}\right]\left[\begin{array}{c}
\mathbf{u}(t) \\
\mathbf{u}_{p}(t)
\end{array}\right] \\
\mathbf{y}(t) & =C_{2}^{g} \mathbf{x}^{g}(t)+D_{21}^{g} \mathbf{w}(t) \\
& +\left[\begin{array}{ll}
D_{22}^{g} & D_{22}^{g}
\end{array}\right]\left[\begin{array}{c}
\mathbf{u}(t) \\
\mathbf{u}_{p}(t)
\end{array}\right]
\end{aligned}\right.
$$

where $E^{g}$ is now invertible. As now $\mathbf{G}(\tilde{\mathbf{H}}) \in \mathcal{L}_{\infty}$, solving an $\mathcal{H}_{\infty}$ control design problem is now well posed. The control objective thus consists in finding the (sub)optimal controller $\tilde{\mathbf{K}}^{\star}(s)$, mapping $\mathbf{y}(t)$ to $\mathbf{u}(t)$ and $\mathbf{u}_{p}(t)$, such that (11) is solved

$$
\tilde{\mathbf{K}}^{\star}:=\arg \min _{\tilde{\mathbf{K}} \in \mathcal{K} \subseteq \mathcal{H}_{\infty}}\left\|\mathcal{F}_{l}(\mathbf{G}(\tilde{\mathbf{H}}), \tilde{\mathbf{K}})\right\|_{\mathcal{H}_{\infty}},
$$

with the additional constraint that the controller $\tilde{\mathbf{K}}^{\star}(s)$ second output set are the derivative of the first one, as imposed in (9). Such a constraint is hard to achieve in a numerical way, this is why in addition, we suggest a specific controller structure that will enable to easily compute the output derivative set. Before detailing such a structure, let us introduce the alternate control problem invoked latter as

$$
\tilde{\mathbf{K}}_{\mathbf{u}}^{\star}:=\arg \min _{\tilde{\mathbf{K}}_{\mathbf{u}} \in \mathcal{K} \subseteq \mathcal{H}_{\infty}}\left\|\mathcal{F}_{l}\left(\mathbf{G}_{\mathbf{u}}(\tilde{\mathbf{H}}), \tilde{\mathbf{K}}_{\mathbf{u}}\right)\right\|_{\mathcal{H}_{\infty}},
$$

where $\mathbf{G}_{\mathbf{u}}(\tilde{\mathbf{H}})$ and $\tilde{\mathbf{K}}_{\mathbf{u}}$ have the same realization as $\mathbf{G}(\tilde{\mathbf{H}})$ and $\tilde{\mathbf{K}}$, respectively, but with one single control input signal, being $\mathbf{u}(t)$ and omitting $\mathbf{u}_{p}(t)$.

\section{Controller structured form}

Let us now define the $\tilde{\mathbf{K}}_{\mathbf{u}} \subseteq \mathcal{K}_{\mathbf{u}}$ controller with realization $\tilde{\mathcal{S}}_{K_{\mathrm{u}}}$ embedding the following structure:

$$
\left\{\begin{aligned}
\dot{\mathbf{x}}_{K}(t)= & {\left[\begin{array}{cccc}
0 & e_{1} & 0 & 0 \\
0 & 0 & \ddots & 0 \\
0 & & 0 & e_{n_{K}-1} \\
a_{1} & \ldots & a_{n_{K}-1} & a_{n_{K}}
\end{array}\right] \mathbf{x}_{K}(t) } \\
+ & {\left[\begin{array}{c}
b_{1} \\
\vdots \\
b_{n-1} \\
b_{n_{K}}
\end{array}\right] \mathbf{y}(t) } \\
\mathbf{u}(t) & =\left[\begin{array}{llll}
c_{1} & \cdots & c_{n_{K}-1} & 0
\end{array}\right] \mathbf{x}_{K}(t)
\end{aligned}\right.
$$

This controller structure is then used to solve (12). Interestingly, thanks to such a realization, as a direct consequence, one can directly access to $\dot{\mathbf{u}}(t)$ by a structural derivation as follows.

$$
\begin{aligned}
\dot{\mathbf{u}}(t) & =\left[\begin{array}{llll}
0 & c_{1} e_{1} & \ldots & c_{n_{K}-1} e_{n_{K}-1}
\end{array}\right] \mathbf{x}_{K}(t) \\
& +\left[\begin{array}{lll}
c_{1} & \ldots & c_{n_{K}-1}
\end{array}\right]\left[\begin{array}{c}
b_{1} \\
\vdots \\
b_{n_{K}-1}
\end{array}\right] \mathbf{y}(t) .
\end{aligned}
$$

Then, the complete controller $\mathbf{K}$ with realization $\mathcal{S}_{K}$, including the input derivative $\dot{\mathbf{u}}(t)$, which solves problem (11), is simply given as

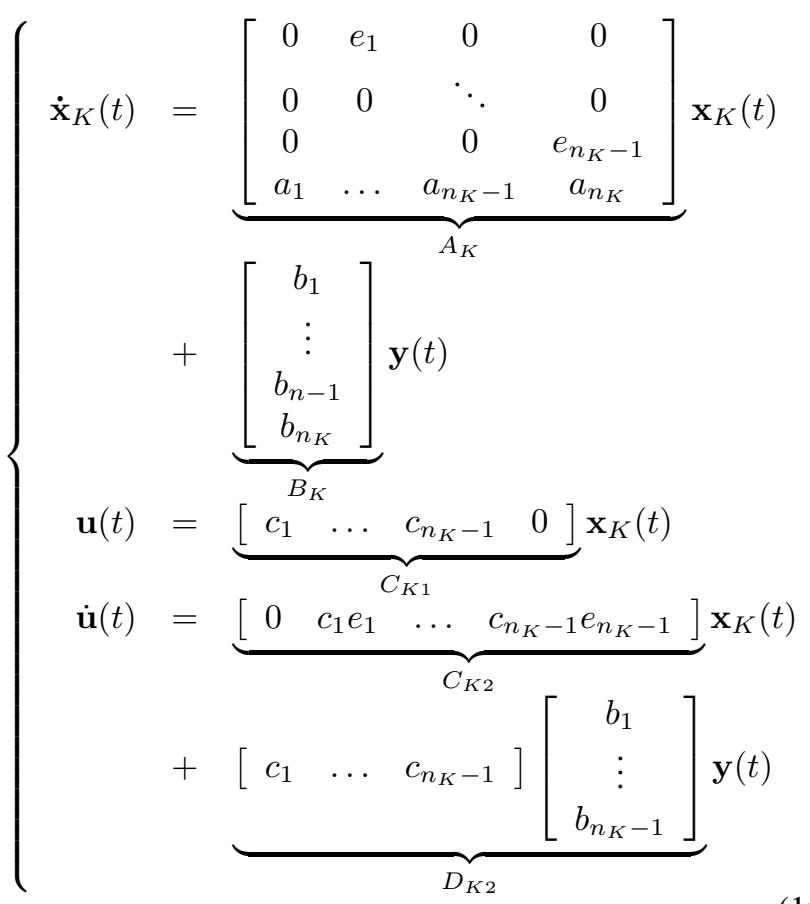

Then, controller (15) provides the exact solution of the input derivative index-2 $\mathcal{H}_{\infty}$ control problem (6). Now let us formulate the following main result related to the given procedure and obtained controller.

Result 1 (Controller rank and eigenvalues): Given the controller realization (15), which reads

$$
\begin{aligned}
\mathcal{S}_{K}: & \left(I_{n_{K}}, A_{K}, B_{K},\left[\begin{array}{ll}
C_{K 1}^{T} C_{K 2}^{T} \\
:
\end{array}\right]^{T},\left[\begin{array}{ll}
0 & D_{K 2}
\end{array}\right]^{T}\right) \\
: & \left(I_{n_{K}}, A_{K}, B_{K}, C_{K}, D_{K}\right)
\end{aligned}
$$

then, the $\left(I_{n_{k}}, A_{K}\right)$ pencil embeds finite eigenvalues only and $\operatorname{rank}\left(A_{K}\right)=\operatorname{dim}\left(A_{K}\right)$, i.e. the rank is non deflective.

Proof: The proof immediately comes from the rank of the dynamical matrix $A_{K}$ in (15), which is full by imposing $\left\{e_{i}\right\}_{i=1}^{n_{K}-1} \neq 0$ and $a_{1} \neq 0$, which is classically selected when optimizing a given controller.

\section{Alternate (naive) solution}

Let us now address the case where the above procedure has not been deployed. First let remind that addressing (6) (i.e. using the structured controller $\mathcal{H}_{\infty}$ approach) using the index-2 DAE model is not possible as it, due to the polynomial part, rendering the model $\notin \mathcal{L}_{\infty}$. A naive approach might be to use the $\mathbf{u}(t)$ control input only, and to synthesize the controller $\tilde{\mathbf{K}}^{\star}$. Then, the derivative control signal term can be simply obtained by mathematical derivation of the $\tilde{\mathbf{K}}^{\star}$ controller as

$$
\mathbf{u}_{p}(s)=s \mathbf{u}(s) .
$$

As a consequence, the resulting generated $\mathcal{S}_{K}$ controller embedding the input derivative $\dot{\mathbf{u}}(t)$ is now equipped with 
DAE realization $\mathcal{S}_{K}$ (similar to the one in (2) or (3)), as

$$
\left\{\begin{array}{c}
E_{K} \dot{\mathbf{x}}_{K}(t)=A_{K} \mathbf{x}_{K}(t)+B_{K} \mathbf{y}(t) \\
{\left[\begin{array}{c}
\mathbf{u}(t) \\
\dot{\mathbf{u}}(t)
\end{array}\right]=C_{K} \mathbf{x}_{K}(t)+D_{K} \mathbf{u}(t)}
\end{array}\right.
$$

where the $\left(E_{K}, A_{K}\right)$ pencil comprises at least one eigenvalue in $\infty$. Moreover, as the resulting controller $\mathcal{S}_{K}$ is now descriptor, its time-domain resolution imposes some algebraic equations and possibly impulsive modes. Therefore, after a discretization step for implementation issues, such form might be complex, if not impossible to solve in a realtime context. Indeed, such a controller would result in both difference and algebraic equations.

\section{Aeroelastic Discrete GUST-ORIENTED AIRCRAFT LOAD CONTROL APPLICATION}

\section{A. Description of the set-up}

Let us now apply the proposed methodology on a generalized state-space aeroservoelastic model, represented in Figure 1, known as the FERMAT model, which is used to design load alleviation controllers.

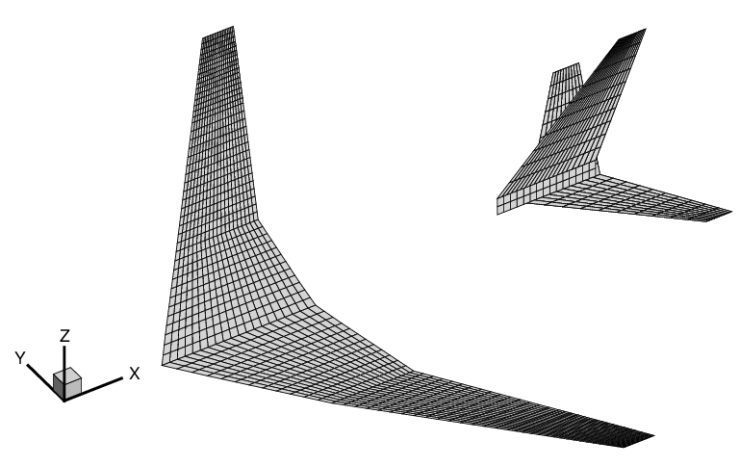

Fig. 1. FERMAT aircraft model.

This latter has been obtained based on tangential interpolation method (see [11] for details). In brief, the resulting DAE system may be reduced to a set of ODE by residualization of the non-proper part of the transfer function matrix. The generalized state-space is of minimal order and allows the application of the force summation method (FSM) for the aircraft loads recovery. Compared to the classical rational function approximation (RFA) approach, the FSM provides a minimal order realization with exact interpolation of the unsteady aerodynamic forces in tangential directions, avoiding any selection of poles (lag states). Then, to complete the model, the polynomial part is added and the proposed aeroservoelastic model of minimal order is then given as explained in (2). One main objective when using this model is then to design active feedback control laws using the movable surfaces (here a lumped input aileron is used) to alleviate the loads, especially at the wing root level, in response to vertical gusts displacement.

\section{B. Control construction and numerical results}

Based on the descriptor model with polynomial part $\mathbf{H}$, with DAE realization $\mathcal{S}$ of dimension $n=168$ (embedding $E$ matrix rank of $r=166$ and non-null $D$ matrix), as detailed in [11], we can construct the canonical form and obtain

$$
N=\left[\begin{array}{ccc}
0 & 1 & 0 \\
0 & 0 & 0 \\
0 & 0 & 0
\end{array}\right] \in \mathbb{R}^{n_{2} \times n_{2}}
$$

with $n_{1}=165$ finite dynamic modes and $n_{2}=3$. Then, based on (19), the nilpotency degree of $N$ is equal to $k=2$ (the model is then an index- 2 one.). Then, the model counts $r-n_{1}=1$ infinite dynamic impulsive mode and $n-r=2$ non dynamic modes. The impulsive mode comes from the polynomial part $\mathbf{H}_{r}$ and the non-dynamic modes come from the direct feed-through term $D$ plus the polynomial part.

The input derivative model $\tilde{\mathcal{S}}$ as in (8) may then be considered instead, and (12) is solved for both the proposed (structured) approach and the alternate naive way (which does not exploits the proposed structured control law). Then, two $\tilde{\mathbf{K}}_{\mathbf{u}}^{\star}$ controllers of dimension $n_{K}=7$ are obtained. The one input two outputs $(\mathbf{u}(t)$ and $\dot{\mathbf{u}}(t)) \mathbf{K}^{\star}$ controllers are derived using the proposed structural derivation, and the Laplace derivation as in (17). The resulting, controllers Bode gain responses are shown on Figure 2.
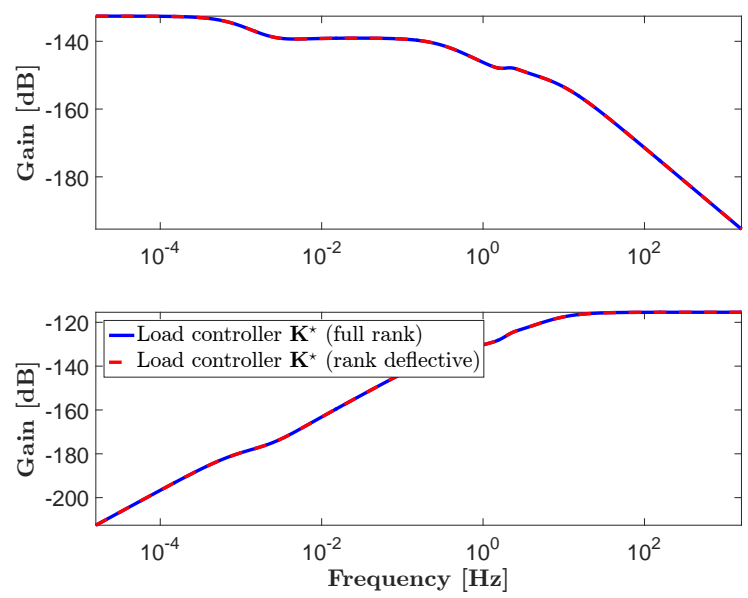

Fig. 2. Bode plot of the proposed full rank approach (solid blue) and the (naive) rank deflective one (dashed red) controllers. Transfer form $\mathbf{y}(t)$ to $\mathbf{u}(t)$ (top) and to $\dot{\mathbf{u}}(t)$ (bottom).

Based on Figure 2, the main element to consider is the fact that the second output clearly is the derivative of the first one for both approaches. Still, as the main remark stated in Result 1, when evaluating the rank and dimensions of the obtained realizations of the naive and the proposed structured approach of $\mathbf{K}^{\star}$ (15), the following results are obtained (for $\left.n_{K}=7\right)$ : 
- the naive way leads to $\operatorname{dim}\left(E_{k}\right)=\operatorname{dim}\left(A_{k}\right)=9$ but with $\operatorname{rank}\left(E_{k}\right)=8$ and $\operatorname{rank}\left(A_{k}\right)=9$ and two infinite eigenvalues.

- the newly proposed approach leads to $\operatorname{dim}\left(E_{k}\right)=$ $\operatorname{dim}\left(A_{k}\right)=7$ and $\operatorname{rank}\left(E_{k}\right)=\operatorname{rank}\left(A_{k}\right)=7$, with finite eigenvalues only.

Consequently, while the classical approach would lead to a controller impossible to implement in a real-time set-up, the proposed approach, simply is a classical linear ODE and provides the same performances. Moreover, the Laplace derivation obviously led to a non-minimal McMillian degree of the controller.

To complete the example, the obtained closed-loop performances from the gust input disturbance $\mathbf{w}(t)$, to the wing root bending moment (performance output $\mathbf{z}(t)$ ) obtained with the proposed controller, are shown on Figure 3 for the Bode gain response and on Figure 4, for the time-domain response to a classical 1-cosine gust profile (traditionally used by authorities to evaluate the load impact).

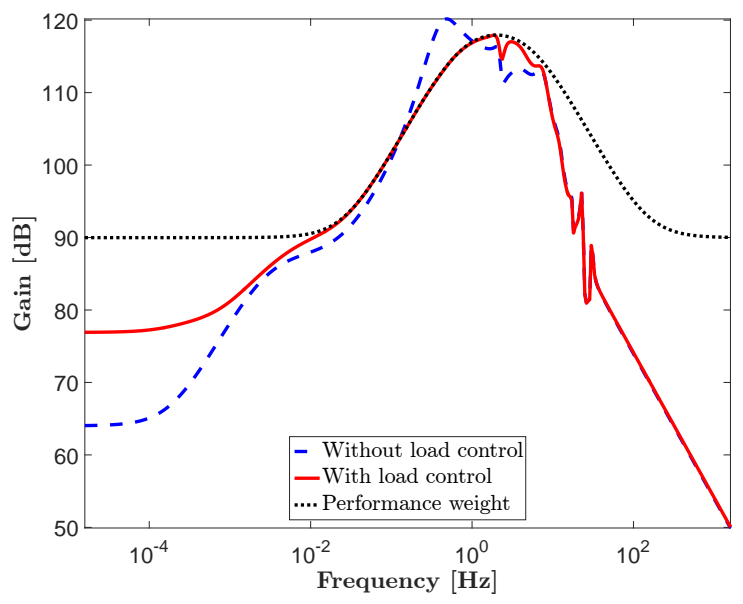

Fig. 3. Bode diagram of the performance transfer form gust to wing root bending moment in with (solid red) and without (dashed blue) load controller. Weighting function (black dotted).

By paying attentions to Figures 3 and 4, and without lack of generality, it is clear that the proposed load control design is appropriate in reducing the impact of the gust on the loads, and its time-domain simulation applicable, thanks to the ODE structure.

\section{CONCLUSIONS AND PERSPECTIVES}

In this paper we proposed a simple but yet effective approach to design a reduced order controller that can be applied to input derivative-like SISO index-2 descriptor models. The approach is basically grounded on an appropriate structure of the controller and a model reformulation, allowing to treat the DAE problem as a standard ODE one, on which $\mathcal{H}_{\infty}$ control method can be applied. The methodology takes advantage of the structured $\mathcal{H}_{\infty}$-norm oriented controller optimization tools in order to seek for a control law on which a simple structural derivation can be applied without any

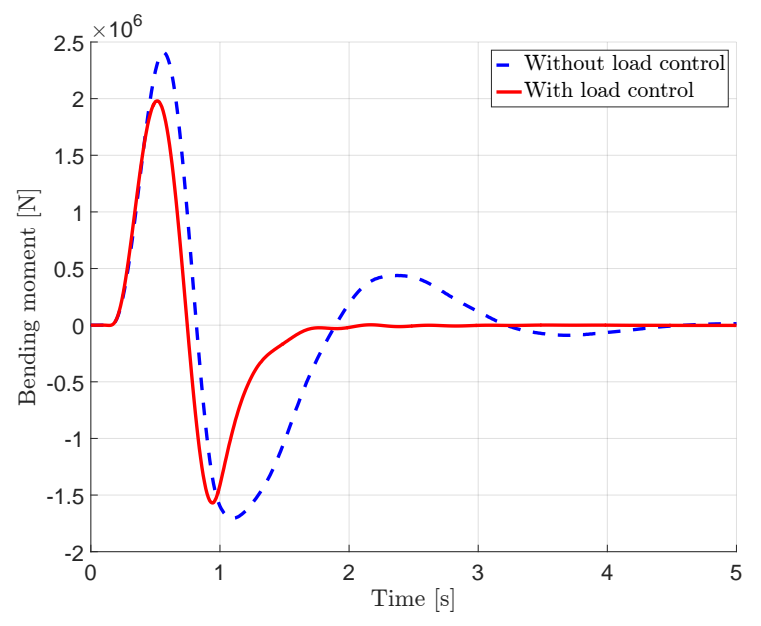

Fig. 4. Wing root bending moment in response to a discrete gust with (solid red) and without (dashed blue) load controller.

numerical loss. The overall approach has been numerically validated on a complex high order flexible aircraft model which naturally embeds an input derivative action leading to a transfer function with a first order polynomial term.

The present paper provides a solution for the first derivative case, but extension to the higher derivatives may straightforwardly obtained following the same path. These cases might appear in many structural engineering applications.

\section{ACKNOWLEDGMENT}

This work has been funded within the frame of the Joint Technology Initiative JTI Clean Sky 2, AIRFRAME Integrated Technology Demonstrator platform AIRFRAME ITD (contract N. CSJU-CS2-GAM-AIR-2014-15-01 Annex 1, Issue B04, October 2nd, 2015) being part of the Horizon 2020 research and Innovation framework program of the European Commission

\section{REFERENCES}

[1] J. Sjobergl, "Descriptor systems and control theory," Master's thesis, Linkoping Universitet, Linkoping Universitet, April 2005.

[2] J. Partington, Linear operators and linear systems: an analytical approach to control theory. Cambridge University Press, 2004, vol. 60.

[3] A. C. Antoulas, Approximation of Large-Scale Dynamical Systems. Philadelphia: Advanced Design and Control, SIAM, 2005.

[4] J.-F. Magni, "Linear fractional representation toolbox for use with matlab," Onera, Toulouse, France, Tech. Rep., 2006. [Online]. Available: http://w3.onera.fr/smac/

[5] K. Zhou and J. C. Doyle, Essentials Of Robust Control. Prentice Hall, 1997.

[6] P. Apkarian and D. Noll, "Nonsmooth $\mathcal{H}_{\infty}$ Synthesis," IEEE Transaction on Automatic Control, vol. 51, no. 1, pp. 71-86, January 2006.

[7] T. Klimmek, "Parametric set-up of a structural model for FERMAT configuration aeroelastic and loads analysis," Journal of Aeroelasticity and Structural Dynamics, vol. 3, no. 2, 2014.

[8] C. Poussot-Vassal, D. Quero, and P. Vuillemin, "Data-driven approximation of a high fidelity gust-oriented flexible aircraft dynamical model," in Proceedings of the IFAC Mathematical Modelling, Vienna, Austria, February 2018.

[9] A. Rehm and F. Allgower, " $\mathcal{H}_{\infty}$ control of descriptor systems with high index," in Proceedings of the IFAC World Congress, Beijing, China, 1999, pp. 1606-1611. 
[10] P. Losse and T. Reis, " $\mathcal{H}_{\infty}$-Control for Descriptor Systems - A Structured Matrix Pencils Approach," in Proceedings of the 48th IEEE Conference on Decision and Control, Shanghai, China, December 2009, pp. 103-108.

[11] D. Quero, P. Vuillemin, and C. Poussot-Vassal, "A Generalized StateSpace Aeroservoelastic Model based on Tangential Interpolation," Aerospace, 2019. 